\title{
Evaluation and Identification of Promising Lines for Rain Fed Conditions in Indian mustard (Brassica juncea L.)
}

\author{
V.V. Singh*, Pankaj Garg, M.L. Meena, H.S. Meena, Priyamedha, \\ Arun Kumar and P. K. Rai \\ ICAR-Directorate of Rapeseed-Mustard Research, Sewar, Bharatpur-321 303 (Rajasthan), \\ India \\ *Corresponding author
}

\section{A B S T R A C T}

One hundred twenty five advanced lines $\left(\mathrm{F}_{6}\right)$ with four check varieties were evaluated in an augmented randomized complete block design for 13 different morphological traits under irrigated $\left(\mathrm{E}_{1}\right)$ and water stressed $\left(\mathrm{E}_{2}\right)$ conditions with five blocks during rabi 201516. Heritability was high $(>50 \%)$ for all these characters except for test weight and oil

Keywords

Heritability,

Correlation, DSI,

Rainfed, Indian

mustard

Article Info

Accepted:

26 November 2018

Available Online:

10 December 2018 content under $\mathrm{E}_{1}$ condition and for fruit zone length and oil content under $\mathrm{E}_{2}$ condition. The overall mean performance of progenies was comparatively higher in irrigated environment for plant height $(\mathrm{PH})$, primary branches (PB), secondary branches (SB), fruiting zone length (FZL), main shoot length (MSL), siliquae on main shoot (SMS), siliquae per plant (SP), siliquae length (SL), seeds per siliquae (SPS), biological yield (BY) and seed yield per plant (SY). Seed yield per plant had positively and significantly correlated with PH (0.222), PB (0.641), SB (0.756), FZL (0.472), MSL (0.374), SMS (0.269), SP (0.722), SL (0.216) and BY 0.872) under irrigated condition while under rainfed condition seed yield per plant had positively and significantly correlated with all characters studied except oil content. On the basis of correlation study, it is suggested that PH, PB, SB, FZL, MSL, SMS, SP, SL and BY should be considered in selection programme for yield improvement in the segregating material generated by using selected advance lines. The values of DSI ranged from -1.544 (DRMR-1616-68-30-61) to 1.710 (DRMR-1722-45). The genotypes DRMR-1721-24, DRMR-1616-68-30-61 and DRMR1676-71-32 had lower DSI values thus rated as drought tolerant.

\section{Introduction}

Moisture stress during the crop growth is one of the main constraints for sustainable mustard productivity, especially in rainfed areas. Growth and seed yield production of Brassica species have greatly decreased due to drought conditions. In India, Brassica juncea is a predominant species which accounts for nearly $80 \%$ of the oilseed brassicas. The crop is mainly grown for its oil which is largely used in cooking and frying purposes. Oilseed cake or meal which is a byproduct during the extraction of oil from the seeds, is an important source of protein feed for animals while the leaves of the plant are used as 
vegetable as well as fodder for cattle. The magnitude of genetic parameters of seed yield and its component traits for different conditions is essential for an effective breeding programme. The genetic variability is the basic requirement for making progress in crop breeding. For improvement in yield, it would be desirable to understand the nature and magnitude of associations amongst yield and its component traits. The correlation coefficient is a measure of the degree of association between two traits worked at the same time (Hayes et al., 1955). Based upon genotypic and phenotypic correlations, the breeder would be able to decide the breeding method to be used to exploit the desirable and break the undesirable associations. Drought Susceptibility Index (DSI) is calculated for each genotype as a criterion of drought tolerance. Genotypes with the lowest DSI, particularly for seed yield at both locations, would serve as useful donors in the breeding programme for improving the drought tolerance of existing Indian mustard cultivars.

\section{Materials and Methods}

The material for present investigation consisted of 125 advanced progenies $\left(\mathrm{F}_{6}-\mathrm{F}_{7}\right)$ of Indian mustard selected on the basis of high yield under rainfed conditions in previous generation. The experiment was carried out at research farm of ICAR-Directorate of Rapeseed Mustard Research, Sewar, Bharatpur during 2015-16.

Experiment was done in an augmented block design (Fedrer, 1956) under normal $\left(\mathrm{E}_{1}\right)$ and water-stressed $\left(\mathrm{E}_{2}\right)$ conditions. The material was divided into five blocks consisting of 25 progenies and four check varieties in each block, namely, DRMRIJ-31, RH-749, NRCDR-02 and NRCHB-101. In each block, progenies and check varieties were sown in two row plots of five meter length, spaced 30 $\mathrm{cm}$ apart with plant-to-plant spacing of $10 \mathrm{~cm}$ achieved by thinning after 15-20 days of sowing. Border effect was removed by taking observations on middle plants in a row. Waterstressed $\left(\mathrm{E}_{2}\right)$ was grown as rainfed experiment and only pre-sowing irrigation was given, while normal $\left(E_{1}\right)$ environment was irrigated twice during cropping season, one at 35 days after sowing and another at 70 days after sowing. Observations were recorded on 13 morphological characters viz., plant height, primary branches per plant, secondary branches per plant, fruiting zone length, main shoot length, siliqua on main shoot, siliquae per plant, siliqua length, seeds per siliqua, biological yield, seed yield per plant, test weight and oil content. The mean data were subjected to analysis of variance (Fedrer, 1956) using SPAD (Abhishek et al., 2004) software. Genetic parameters and simple correlations in all possible combinations were worked out as per standard procedure (Burton, 1952; Johnson et al., 1955). The relative drought tolerance of genotypes was quantified with respect to seed yield through DSI according to Fischer and Maurer (1978).

\section{Results and Discussion}

In irrigated environment as compared to rainfed condition for all characters studied overall mean performance of progenies was comparatively higher except for test weight and oil content (Table 1). For test weight and oil content, mean performance was slightly higher in rainfed condition as compared to irrigated conditions. Kumawat et al., (1997) described the decreased in translocation of assimilates and growth substances, loss of turgidity might be affect on mean performance of progenies under drought situations for most of the traits. In Brassica juncea, Singh and Choudhary (2003) and Chauhan et al., (2007) reported up to $60 \%$ yield reductions under rainfed environments.

Under irrigated condition, progenies showed significant differences for all character studied except for fruit zone length and oil content 
while progenies differed significantly for all character studied except for oil content under rainfed conditions. This indicates that material has sufficient variability for these traits and response to selection may be expected in the breeding programme for irrigated as well as rainfed conditions.

Generally magnitude of phenotypic coefficient of variation (PCV) higher than magnitude of genotypic coefficient of variation (GCV). It was observed that siliqua per plant followed by biological yield and seed yield per plant under irrigated condition while under irrigated condition siliqua per plant followed by biological yield and secondary branches exhibited comparatively higher estimates of genotypic as well as phenotypic coefficient of variation (Table-1). It indicated that simple selection for these characters might be advantageous in particular condition. Since, in augmented design only the error variance of check varieties could be subtracted from the variance of genotypes a portion of it may be confounded with the genotypic variance used for calculating the heritability. The estimates of heritability in present investigation were of higher magnitude $(>50 \%)$ for all the characters with significant variability except for test weight and oil content under irrigated condition and except for fruit zone length and oil content under rainfed condition. These result was also confirmed by Singh et al., (2009) and Meena et al., (2008).

The genetic advance was highest for siliqua per plant followed by biological yield and plant height under irrigated conditions whereas, under rainfed condition siliqua per plant followed by plant height and biological yield showed higher estimates of genetic advance. These findings indicate that there is good scope for development of genotypes having more number of siliquae per plant, biological yield, increased fruit zone length, higher number of siliqua on main shoot and which would perform better in irrigated conditions. Similarly, there is enough scope for development of promising genotypes having increased siliquae per plant, biological yield and more number of siliquae on main shoot for water stress conditions. High heritability with high genetic advance were observed for siliqua per plant, biological yield, fruit zone length and siliqua on main shoot under irrigated conditions while siliqua per plant, biological yield, siliqua on main shoot and main shoot length under rainfed. This indicates that selection will be more effective for these characters as compared to others. Similar reports of high heritability with high genetic advance for these characters (Patel et al., 2006 and Singh et al., 2011).

Simple correlations were estimated between seed yield and component traits in irrigated as well as rainfed conditions (Table 2). Under irrigated conditions, seed yield per plant was positively and significantly correlated with plant height $(0.222)$, primary branches per plant (0.641), secondary branches per plant (0.756), fruiting zone length $(0.472)$, main shoot length (0.374), siliqua on main shoot (0.269), siliquae per plant (0.722), siliqua length (0.216) and biological yield (0.872) while under rainfed conditions, seed yield per plant was significantly and positively correlated with plant height $(0.455)$, primary branches per plant (0.465), secondary branches per plant (0.651), fruiting zone length (0.481), main shoot length (0.474), siliqua on main shoot $(0.551)$, siliquae per plant (0.611), siliqua length (0.310), seeds per siliqua (0.339), biological yield (0.688) and test weight $(0.267)$. These results were in agreement with the earlier reports of Kardam and Singh (2005), Meena et al., (2008) and Singh et al., (2009, 2011 and 2015). Therefore, for efficient use of scarce water resource high yielding genotypes can be developed with long siliqua length, more seeds per siliqua, test weight and fast growing seedling traits. Under 
irrigated conditions, seed yield per plant was negatively and significantly correlated with oil content (0.092). It was also noted that characters which exhibited positive association with seed yield per plant also exhibited positive association among themselves thus these characters could be simultaneously improved to increase the seed yield. Association between some characters was non significant which implies that the two variables are not linearly related on these two may be related but in a non linear fashion (Gomez and Gomez, 1980).

Table.1 Estimates of Mean, Range, GCV, PCV, Heritability (bs) and Genetic advance for seed yield and its components in advanced progenies of Indian mustard under rainfed and irrigated conditions

\begin{tabular}{|c|c|c|c|c|c|c|c|c|}
\hline Trait & Condition & Mean & $\begin{array}{l}\% \text { reduction in } \\
\text { mean values }\end{array}$ & Range & GCV & PCV & $\begin{array}{l}\text { Heritability } \\
\text { (broad sense) }\end{array}$ & $\begin{array}{c}\text { Genetic advance as } \\
\% \text { of mean }\end{array}$ \\
\hline \multirow[t]{2}{*}{ PH } & IR & 192.88 & \multirow[t]{2}{*}{11.42} & $148.20-233.80$ & 11.49 & 11.81 & 94.61 & 31.96 \\
\hline & $\mathbf{R F}$ & 170.85 & & $121.60-202.20$ & 7.94 & 9.91 & 64.25 & 17.14 \\
\hline \multirow[t]{2}{*}{ PB } & IR & 6.21 & \multirow[t]{2}{*}{28.50} & $3.80-11.40$ & 4.65 & 4.70 & 97.81 & 2.36 \\
\hline & $\mathbf{R F}$ & 4.44 & & $2.60-6.80$ & 3.35 & 3.58 & 87.17 & 1.35 \\
\hline \multirow[t]{2}{*}{ SB } & IR & 10.75 & \multirow[t]{2}{*}{64.47} & $3.60-28.00$ & 11.38 & 11.44 & 98.85 & 7.64 \\
\hline & $\mathbf{R F}$ & 3.82 & & $0.20-8.80$ & 9.29 & 9.54 & 94.70 & 3.63 \\
\hline \multirow[t]{2}{*}{ FZL } & IR & 84.47 & \multirow[t]{2}{*}{17.49} & $23.40-104.40$ & 10.72 & 10.82 & 98.14 & 20.10 \\
\hline & $\mathbf{R F}$ & 69.70 & & $43.60-90.80$ & $*$ & $*$ & $*$ & $*$ \\
\hline \multirow[t]{2}{*}{ MSL } & IR & 77.89 & \multirow[t]{2}{*}{18.65} & $51.20-100.40$ & 7.83 & 8.75 & 79.94 & 12.72 \\
\hline & $\mathbf{R F}$ & 63.36 & & $45.80-92.40$ & 8.26 & 9.76 & 71.58 & 11.45 \\
\hline \multirow[t]{2}{*}{ SMS } & IR & 44.55 & \multirow[t]{2}{*}{17.33} & $33.20-93.00$ & 10.09 & 11.16 & 81.80 & 12.55 \\
\hline & $\mathbf{R F}$ & 36.83 & & $25.40-56.80$ & 9.19 & 9.21 & 99.61 & 11.47 \\
\hline \multirow[t]{2}{*}{ SP } & IR & 272.76 & \multirow[t]{2}{*}{47.98} & $102.00-484.80$ & 43.82 & 43.84 & 99.94 & 149.05 \\
\hline & $\mathbf{R F}$ & 141.90 & & $56.60-299.40$ & 35.12 & 36.75 & 91.37 & 82.39 \\
\hline \multirow[t]{2}{*}{ SL } & IR & 4.28 & \multirow[t]{2}{*}{3.04} & $3.38-5.04$ & 1.17 & 1.43 & 67.82 & 0.41 \\
\hline & $\mathbf{R F}$ & 4.15 & & $3.28-5.21$ & 1.32 & 1.56 & 71.29 & 0.47 \\
\hline \multirow[t]{2}{*}{ SPS } & IR & 14.13 & \multirow[t]{2}{*}{7.64} & $10.88-18.40$ & 3.13 & 3.16 & 97.87 & 2.39 \\
\hline & $\mathbf{R F}$ & 13.05 & & $9.88-15.92$ & 2.49 & 2.97 & 70.32 & 1.55 \\
\hline \multirow[t]{2}{*}{ BY } & IR & 76.21 & \multirow[t]{2}{*}{52.29} & $38.00-150.00$ & 24.32 & 24.54 & 98.23 & 43.34 \\
\hline & $\mathbf{R F}$ & 36.36 & & $15.00-65.00$ & 13.68 & 16.56 & 68.26 & 14.04 \\
\hline \multirow[t]{2}{*}{ SY } & IR & 15.93 & \multirow[t]{2}{*}{56.18} & $5.09-35.72$ & 14.18 & 14.77 & 92.20 & 11.20 \\
\hline & $\mathbf{R F}$ & 6.98 & & $1.92-15.44$ & 8.47 & 9.48 & 79.90 & 4.12 \\
\hline \multirow[t]{2}{*}{ TW } & IR & 4.73 & \multirow[t]{2}{*}{-0.21} & $3.02-6.73$ & 1.80 & 2.76 & 42.78 & 0.53 \\
\hline & $\mathbf{R F}$ & 4.74 & & $3.02-6.93$ & 2.63 & 2.82 & 87.04 & 1.10 \\
\hline \multirow[t]{2}{*}{ Oil } & IR & 42.00 & \multirow{2}{*}{$\begin{array}{c}- \\
1.09\end{array}$} & $40.10-44.21$ & $*$ & $*$ & $*$ & $*$ \\
\hline & $\mathbf{R F}$ & 42.46 & & $40.55-43.96$ & $*$ & $*$ & $*$ & $*$ \\
\hline
\end{tabular}

* Mean sum squares were non-significant for these characters; hence genetic parameters were not calculated

$\mathrm{I}=$ Irrigated, RF=Rainfed, $\mathrm{PH}=$ Plant height, $\mathrm{PB}=$ Primary branches per plant, $\mathrm{SB}=$ Secondary branches per plant, FZL=Fruiting zone length, MSL=Main shoot length, SMS=Siliquae on main shoot, SP=Siliquae per plant, SL=Siliquae length, SPS=Seeds per siliqua, BY=Biological yield, $\mathrm{SY}=$ Seed yield per plant, $\mathrm{TW}=$ test weight 
Table.2 Correlation coefficients between different characters in advanced progenies of Indian mustard under irrigated and rainfed conditions

\begin{tabular}{|c|c|c|c|c|c|c|c|c|c|c|c|c|c|c|}
\hline Trait & Condition & $\mathrm{PH}$ & PB & SB & FZL & MSL & SMS & SP & SL & SPS & BY & SY & TW & Oil \\
\hline \multirow[t]{2}{*}{$\mathrm{PH}$} & IR & 1.000 & & & & & & & & & & & & \\
\hline & RF & 1.000 & & & & & & & & & & & & \\
\hline \multirow[t]{2}{*}{ PB } & IR & $0.279^{* *}$ & 1.000 & & & & & & & & & & & \\
\hline & RF & $0.375^{* *}$ & 1.000 & & & & & & & & & & & \\
\hline \multirow[t]{2}{*}{ SB } & IR & $0.114 * *$ & $0.804 * *$ & 1.000 & & & & & & & & & & \\
\hline & $\mathbf{R F}$ & $0.380^{* *}$ & $0.582 * *$ & 1.000 & & & & & & & & & & \\
\hline \multirow[t]{2}{*}{ FZL } & IR & $0.484 * *$ & $0.297 * *$ & $0.355^{* *}$ & 1.000 & & & & & & & & & \\
\hline & RF & $0.621 * *$ & $0.415^{* *}$ & $0.533 * *$ & 1.000 & & & & & & & & & \\
\hline \multirow[t]{2}{*}{ MSL } & IR & $0.447 * *$ & $0.190 * *$ & $0.305 * *$ & $0.624 * *$ & 1.000 & & & & & & & & \\
\hline & RF & $0.543^{* *}$ & $0.182 * *$ & $0.437 * *$ & $0.666^{* *}$ & 1.000 & & & & & & & & \\
\hline \multirow[t]{2}{*}{ SMS } & IR & $0.302 * *$ & $0.159 * *$ & $0.224 * *$ & $0.295 * *$ & $0.465^{* *}$ & 1.000 & & & & & & & \\
\hline & RF & $0.491 * *$ & $0.370^{* *}$ & $0.532 * *$ & $0.608 * *$ & $0.729 * *$ & 1.000 & & & & & & & \\
\hline \multirow[t]{2}{*}{ SP } & IR & $0.248^{* *}$ & $0.730 * *$ & $0.831^{* *}$ & $0.453 * *$ & $0.416^{* *}$ & $0.283^{* *}$ & 1.000 & & & & & & \\
\hline & $\mathbf{R F}$ & $0.434^{* *}$ & $0.695 * *$ & $0.684 * *$ & $0.587 * *$ & $0.450 * *$ & $0.584 * *$ & 1.000 & & & & & & \\
\hline \multirow[t]{2}{*}{ SL } & IR & -0.080 & -0.069 & -0.018 & 0.054 & -0.004 & 0.048 & -0.031 & 1.000 & & & & & \\
\hline & RF & $0.201^{* *}$ & 0.079 & $0.222 * *$ & $0.184 * *$ & $0.253^{* *}$ & $0.164 * *$ & $0.146^{* *}$ & 1.000 & & & & & \\
\hline \multirow[t]{2}{*}{ SPS } & IR & 0.084 & -0.028 & -0.007 & -0.034 & -0.011 & 0.063 & -0.074 & $0.351 * *$ & 1.000 & & & & \\
\hline & RF & $0.177^{* *}$ & 0.057 & $0.250 * *$ & $0.238 * *$ & $0.272 * *$ & $0.199 * *$ & $0.206^{* *}$ & $0.544^{* *}$ & 1.000 & & & & \\
\hline \multirow[t]{2}{*}{ BY } & IR & $0.409^{* *}$ & $0.697 * *$ & $0.793 * *$ & $0.522 * *$ & $0.486^{* *}$ & $0.341 * *$ & $0.778^{* *}$ & $0.165^{* *}$ & 0.007 & 1.000 & & & \\
\hline & $\mathbf{R F}$ & $0.525^{* *}$ & $0.365 * *$ & $0.593 * *$ & $0.486 * *$ & $0.489 * *$ & $0.504 * *$ & $0.593 * *$ & $0.426^{* *}$ & $0.295^{* *}$ & 1.000 & & & \\
\hline \multirow[t]{2}{*}{ SY } & IR & $0.222 * *$ & $0.641 * *$ & $0.756^{* *}$ & $0.472 * *$ & $0.374 * *$ & $0.269 * *$ & $0.722 * *$ & $0.216^{* *}$ & 0.058 & $0.872 * *$ & 1.000 & & \\
\hline & RF & $0.455^{* *}$ & $0.465 * *$ & $0.651 * *$ & $0.481 * *$ & $0.474 * *$ & $0.551 * *$ & $0.611^{* *}$ & $0.310^{* *}$ & $0.339 * *$ & $0.688^{* * *}$ & 1.000 & & \\
\hline \multirow[t]{2}{*}{ TW } & IR & $-0.094^{*}$ & $-0.320 * *$ & $-0.257 * *$ & 0.012 & -0.131 & -0.032 & $-0.243 * *$ & $0.411^{* *}$ & $0.087^{*}$ & $-0.158^{* *}$ & 0.044 & 1.000 & \\
\hline & $\mathbf{R F}$ & 0.081 & -0.047 & -0.041 & 0.077 & $0.213^{* *}$ & $0.198 * *$ & $0.106^{*}$ & $0.330^{* *}$ & $0.296^{* *}$ & $0.288^{* *} *$ & $0.267 * *$ & 1.000 & \\
\hline \multirow[t]{2}{*}{ Oil } & IR & $-0.129 * *$ & 0.013 & $0.113^{*}$ & -0.013 & -0.006 & 0.030 & 0.041 & $0.107^{*}$ & 0.035 & 0.047 & $0.092 *$ & $-0.185^{* * *}$ & 1.000 \\
\hline & RF & 0.045 & -0.041 & $-0.098 *$ & 0.016 & $-0.087^{*}$ & $-0.128 * *$ & $-0.132 * *$ & -0.042 & 0.007 & $-0.166 * *$ & $-0.230 * *$ & $-0.367 * *$ & 1.000 \\
\hline
\end{tabular}

$\mathrm{I}=$ Irrigated, $\mathrm{RF}=\mathrm{Rainfed}, \mathrm{PH}=$ Plant height, $\mathrm{PB}=$ Primary branches per plant, $\mathrm{SB}=$ Secondary branches per plant, FZL=Fruiting zone length, MSL=Main shoot length, SMS=Siliquae on main shoot, SP=Siliquae per plant, SL= Siliquae length, SPS=Seeds per siliqua, BY=Biological yield, $\mathrm{SY}=$ Seed yield per plant, $\mathrm{TW}=$ test weight and oil content 
Table.3 Classification of advanced lines of Brassica juncea on the basis of Drought Susceptibility Index (DSI)

\begin{tabular}{|c|c|c|}
\hline DSI Values & Reaction & Progenies \\
\hline$<0.0$ & $\begin{array}{l}\text { Drought } \\
\text { tolerant }\end{array}$ & DRMR-1616-68-30-61, DRMR-1676-71-32, DRMR-1721-24, \\
\hline$>0.0-1.0$ & $\begin{array}{c}\text { Moderately } \\
\text { tolerant }\end{array}$ & $\begin{array}{l}\text { DFS-11, DRMR-1688-55, DRMR-1686-25, YHS-33, DHS-23, YHS-26, DRMR- } \\
\text { 1721-30, DRMR-1680-327-100-73-80, DRMR-1480-12, DRMR-1686-150-21-68, } \\
\text { DRMR-1686-49, DFS-21, DRMR-1724-77, DFS-22, DRMR-1678-26, DRMR- } \\
\text { 1722-40, DRMR-1686-163-23-15-75, DFS-18, YHS-43, DRMR-1724-68, DRMR- } \\
\text { 1686-24, DRMR-1684-57, DRMR-1688-54, DRMR-1721-27, DRMR-1686-38, } \\
\text { DRMR-1722-52, DRMR-1722-42, DRMR-1716-10, DFS-2, DRMR-1688-53, DHS- } \\
\text { 14, DRMR-1686-48, DRMR-1722-50, DRMR-1716-8, DRMR-1716-4, DRMR- } \\
\text { 1717-12, DRMR-1679-22, DRMR-1684-42, DRMR-1692-37, DRMR-1334-17, } \\
\text { DRMR-1686-50, DRMR-1684-58, DFS-1, YHS-113, DRMR-1686-39, DRMR- } \\
\text { 1722-49, DFS-9, DRMR-1334-18, DRMR-1679-21, DRMR-1724-79 }\end{array}$ \\
\hline$>1.0-1.5$ & $\begin{array}{l}\text { Moderately } \\
\text { susceptible }\end{array}$ & $\begin{array}{l}\text { DRMR-1672-5, DRMR-1724-72, DRMR-1722-53, 125, DRMR-1480-1, DHS-29, } \\
\text { DRMR-1722-55, DRMR-1722-41, YHS-57, DRMR-1724-73, DRMR-1686-51, } \\
\text { DHS-28, DRMR-1686-33, DRMR-1688-52, DRMR-1717-15, DHS-24, DRMR- } \\
\text { 1721-22, DRMR-1716-6, 124, DRMR-1724-67, DRMR-1723-58, DRMR-1723-63, } \\
\text { DHS-13, DRMR-1724-65, YHS-44, DRMR-1721-32, DRMR-1716-3, DRMR-1716- } \\
\text { 2, DRMR-1686-34, DRMR-1721-38, DRMR-1721-33, DRMR-1721-29, YHS-36, } \\
\text { DRMR-1686-32, DRMR-1724-74, DRMR-1723-59, DRMR-1721-25, DRMR-1678- } \\
\text { 303-5151-74, DRMR-1721-37, DRMR-1721-23, DRMR-1718-13, DRMR-1717-14, } \\
\text { DRMR-1722-51, DHS-22, DRMR-1721-20, DRMR-1724-64, DRMR-1717-11, } \\
\text { DRMR-1721-31, DRMR-1716-7, DRMR-1566-4, DRMR-1686-31, DRMR-1722- } \\
\text { 43, DRMR-1716-1, DRMR-1679-20, DRMR-1566-58-24-7, DHS-18, DRMR-1721- } \\
\text { 34, DRMR-1722-39, DRMR-1724-66 }\end{array}$ \\
\hline$>1.5$ & susceptible & $\begin{array}{l}\text { DRMR-1686-29, DRMR-1721-26, DRMR-1716-9, DRMR-1722-44, DRMR-1723- } \\
\text { 62, DRMR-1724-71, DRMR-1721-28, DRMR-1721-21, DRMR-1360-11, DRMR- } \\
\text { 1721-36, DRMR-1718-16, DRMR-1686-27, DRMR-1722-45 }\end{array}$ \\
\hline
\end{tabular}

Drought Susceptibility Index (DSI) was calculated for each genotype as a criterion of drought tolerance. The lowest value indicates the highest level of drought tolerance and vice-versa. The values of DSI ranged from 1.544 (DRMR-1616-68-30-61) to 1.710 (DRMR-1722-45) (Table-3). The genotypes DRMR-1616-68-30-61, DRMR-1676-71-32, DRMR-1721-24, had lower DSI values $(<$ or $\sim 0.00$ ), thus rated as drought tolerant. Total 52 genotypes were found moderately drought tolerant $(>0.0-1.0)$ and 59 genotypes were ranked as moderately susceptible (>1.0-1.5) and 13 genotypes were ranked as susceptible (>1.5) (Table 3). Clarke et al., (1984) opined that selection for yield under dry condition should alone be more productive avenue for improvement of drought resistance until more rapid and effective screening procedures could be developed. As DSI is a ratio, a genotype could have lower value of this index even when its mean seed yield under drought condition is significantly lower than better performing genotype (s).

Therefore, genotypes in the present investigation were selected on the basis of high mean seed yield along with lower DSI value. DSI values and seed yield under drought conditions as a selection criterion in Indian mustard used by Singh and Choudhary (2003) and Chauhan et al., (2007). It is pertinent to note that the average performance of this genotype for all the traits was either 
higher than or at par with the mean performance of all the genotypes under drought condition.

\section{References}

Abhishek R, Prasad R and Gupta VK 2004. Computer aided construction and analysis of augmented designs. Journal of Indian Society of Agricultural Statistics. 57: 320-344.

Burton GW. 1952. Quantitative inheritance of grasses. Proceedings of 6th International Grassland Congress. 1: 227-283.

Chauhan JS, Tyagi MK, Kumar A, Nashaat NI, Singh M, Singh NB, Jakhar ML and Welham SJ. 2007. Drought effects on yield and its components in Indian mustard (Brassica juncea L.). Plant Breeding. 126: 399-402.

Clarke J, Townley-Smith TF, McCaaig TN and Green DM. 1984. Growth analysis of spring wheat cultivars of varying drought resistance. Crop Science. 56: 603-626.

Fedrer WT. 1956. Augmented Design. Hawain Planters Record. 20: 191-207.

Fischer RA and Maurer R. 1978. Drought resistance in spring wheat cultivars. 1. Grain yield responses. Australian Journal of Agricultural Research. 29: 897-912.

Gomez KA and Gomez AA. 1980. Statistical Procedures for Agriculture Research. John Wiley and Sons Inc., New York.

Hayes HK, Immer FH and Smith DC. 1955. Methods of Plant Breeding, Mc GrawHill Book Company, Inc. New York. pp: 439.

Johnson HW, Robinson HF and Comstock RE. 1955. Estimate of genetic and environmental variability in soybean. Agronomy Journal. 47: 314-318.

Kardam DK and Singh VV. 2005. Correlation and path coefficient analysis in Indian mustard (Brassica juncea L.) grown under rainfed condition. Journal of Spices and Aromatic Crops. 14: 56-60.

Kumawat BL, Sharma DD and Jat SC. 1997. Effect of Brassinosteroid on yield and yield attributing characteristics under water deficit stress condition in mustard (B. juncea L.). Annals of Biology. 13: 91-93.

Meena SS, Yadav R and Singh VV. 2008. Genetic variability for seed and seedling traits in the advance breeding lines of Indian mustard [Brassica juncea (L.) Czern. \& Coss.]. Seed Research. 36: 152-156.

Patel JM, Patel KM, Patel CJ and Prajapati KP. 2006. Genetic parameter and inter relationship analysis in Indian mustard (Brassica juncea L.). Journal of Oilseed Research. 23: 159-160.

Singh SP and Choudhary AK. 2003. Selection criteria for drought tolerance in Indian mustard (Brassica juncea L.). Indian Journal of Genetics and plant Breeding. 63: 263-264.

Singh VV, Meena ML, Singh BR and Singh D. 2015. Patterns of Genetic Variation, Correlations under Irrigated and Rainfed Conditions in Indian mustard [Brassica juncea (L.)]. Indian Journal of Plant Genetic Resources. 28: 198-204.

Singh VV, Rai PK, Siddiqui SA, Verma V and Yadav R. 2011. Genetic variability and relative drought tolerance in interspecific progenies of Brassica juncea. Agriculture and Biology Journal of North America 2: 34-41.

Singh VV, Singh S, Verma V, Meena SS and Kumar A. 2009. Genetic variability for seedling traits in Indian mustard under moisture stress conditions. Indian Journal of Plant Genetic Resources. 22: 46-49. 


\section{How to cite this article:}

Singh, V.V., Pankaj Garg, M.L. Meena, H.S. Meena, Priyamedha, Arun Kumar and Rai, P.K. 2018. Evaluation and Identification of Promising Lines for Rain Fed Conditions in Indian mustard (Brassica juncea L.). Int.J.Curr.Microbiol.App.Sci. 7(12): 3590-3597. doi: https://doi.org/10.20546/ijcmas.2018.712.406 